\title{
ADEQUAÇÕES NAS PRÁTICAS DOS NOVOS PROCESSOS DE CORTE E DOBRA PARA OTIMIZAR O DESEMPENHO DE AÇOS PLANOS
}

Willy Ank de Morais '

Herbert Christian Borges ${ }^{2}$

\section{Resumo}

Processos de dobra e corte são as principais formas de processamento de chapas metálicas, inclusive aços planos, que são os principais produtos da Usiminas-Cubatão. Cada um dos processos de corte e dobra possui características específicas, que podem ser melhores atendidas com uma chapa de aço mais adequada ou simplesmente por meio de ajustes nesses processos. Neste trabalho estão revisadas condições gerais no que diz respeito a: direção de dobramento; acabamento do corte; elaboração de chanfros e ductilidade do material. Estas considerações são de primordial importância para adequar o aço ao seu uso e assim obter sucesso no desenvolvimento e na utilização de aços planos.

Palavras-chave: Corte; Dobramento; Aço plano; Otimização.

\section{ADEQUACY ON PRACTICE OF NEW PROCESS FOR BENDING AND CUTTING TO OPTIMIZE STEEL SHEET PERFORMANCE}

\begin{abstract}
Bending and cutting are the main way to process metal sheets, including flat steels, which are the chief products of Usiminas-Cubatão. Each type of bending and cutting processes has specific requirements which can be attendant by an adequacy steel sheet or simply by process adjusting. In this work, it is revised the general conditions related to bending direction, cut finishing, chamfering and ductility. These considerations are essential to adequacy of the steel to this use and, thus, obtain success in their development and use.
\end{abstract}

Key words: Cutting; Bending; Flat steel; Optimization.

\section{INTRODUÇÃO}

Processos de corte e dobramento são as operações mais comuns no processamento de metais planos empregados para preparar chapas para as etapas posteriores de processamento e montagem de diversos produtos finais. Tais processos apresentam particularidades que podem influenciar, ou mesmo mascarar, o desempenho do material sendo trabalhado. Assim sendo, este artigo descreve as principais características desses processos, as interferências no processamento de produtos planos de aço e as soluções obtidas simultaneamente por análises laboratoriais e em atividades de assistência técnica realizadas pelos autores.

O dobramento é um processo de conformação plástica gerado pelo surgimento de esforços de tração e compressão simultâneos nos lados contrários da chapa sendo dobrada.(I) Durante o processo, ocorre uma redução de espessura e alongamento na parte sob tração, conforme ilustrado na Figura la. O alongamento máximo pela face externa é o principal fator que limita a operação. A razão entre a espessura inicial da peça $\left(e_{0}\right)$ e $\circ$ raio de dobramento $\left(\mathrm{R}_{\mathrm{i}}\right)$ está diretamente associada com $\circ$ alongamento $\left(\% \Delta \mathrm{e} / \mathrm{e}_{0}\right)$ que $\circ$ material poderá suportar nessa região, conforme se pode observar dos dados experimentais da Figura Ib. Fatores intrínsecos da chapa influenciam a operação de dobramento, como a qualidade das bordas cortadas ou defeitos internos na chapa. Adicionalmente, deve ser considerado o grau de resistência do material, mensurado por meio de ensaios de tração, por exemplo, uma vez que os materiais com maior grau de resistência mecânica tendem a apresentar menor ductilidade. ${ }^{(2,3)}$

\footnotetext{
'Doutorando, Mestre, Engenheiro Metalurgista, Técnico em Metalurgia, Engenheiro de Produto Sênior da Usiminas de Cubatão, Professor da Faculdade de Engenharia da Unisanta e Diretor da divisão técnica "Aplicações de Materiais" da ABM. U2 Ctrl. Prod. Usiminas-Cubatão. Rod. Cônego Domênico Rangoni, s/n, Jardim das Indústrias. E-mail: willyank@unisanta.brou willy.morais@usiminas.com ${ }^{2}$ Mestrando, Engenheiro Metalurgista, Engenheiro de Assistência Técnica Pleno da Usiminas de Cubatão. U2 Ctrl. Prod. Usiminas-Cubatão. Rod. Cônego Domênico Rangoni, s/n, Jardim das Indústrias. E-mail: herbert.borges@usiminas.com
} 
(a)

$$
\% \frac{\Delta e}{e_{0}}=\frac{e_{f}-e_{0}}{e_{0}} \times 100 \%
$$

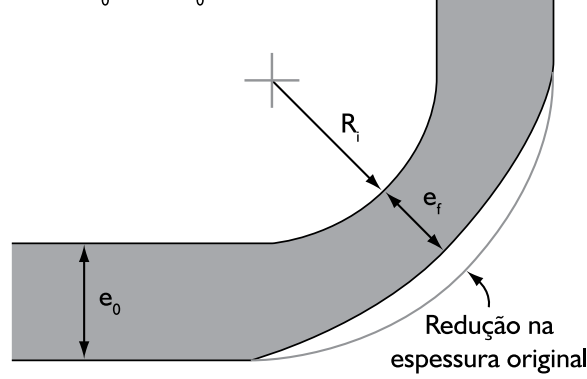

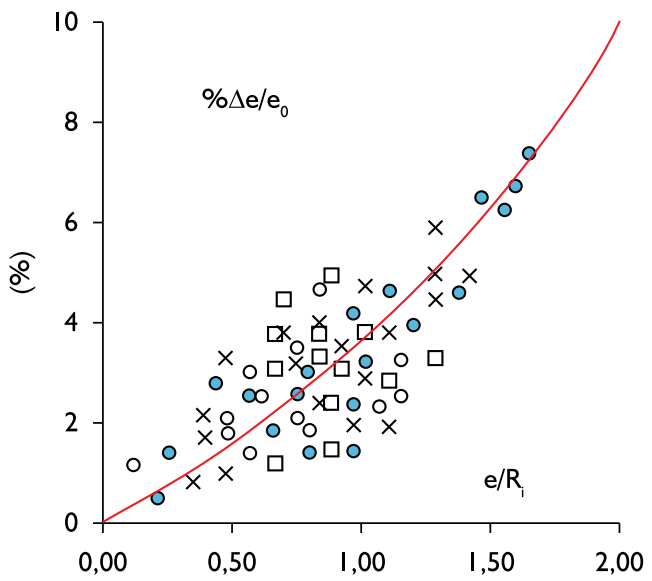
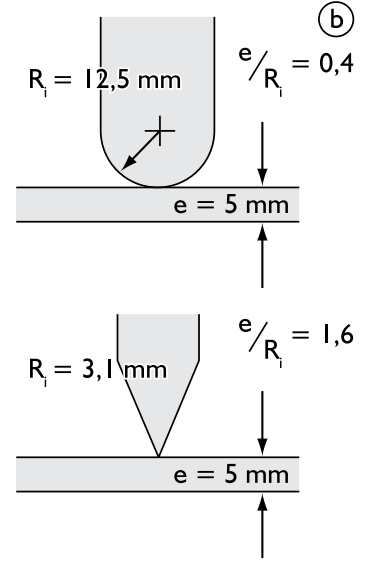

Figura I. a) Alterações geométricas típicas de uma operação de dobramento; e b) Valores obtidos em operações de dobramento entre o raio de dobramento interno $(\mathrm{Ri})$ e a redução de espessura $\left(\Delta \mathrm{e} / \mathrm{e}_{0}\right)$ no dobramento de chapas ${ }^{(1)}:\left(\mathrm{e} / \mathrm{R}_{\mathrm{i}}\right)>\mathrm{I}, 6$ é considerada uma relação crítica.

Por outro lado, muitos processos de corte geralmente envolvem aquecimento e fusão localizada do material, que por vezes levam a alterações microestruturais, que devem ser consideradas. Isto é especialmente verdade no corte de um aço com maior carbono equivalente $\left(C_{e q}\right)$, que pode ser fragilizado quando é austenitizado pelo calor empregado no corte e depois resfriado rapidamente ${ }^{(4)}$ pela extração do calor promovida pelo restante do material que não foi cortado. Mesmo que não ocorra a austenitização do aço, esse tipo de corte pode comprometer o desempenho de ligas nas quais se emprega a precipitação de carbonitretos como mecanismo para regular suas propriedades mecânicas. ${ }^{(5)} \mathrm{Em}$ todos esses casos, a região afetada constitui uma heterogeneidade, que pode gerar problemas no uso posterior da chapa, daí necessitando cuidados especiais.

As operações de corte mecânico normalmente produzem descontinuidades, principalmente rebarbas, na região das bordas e que passam a funcionar como concentradores de tensão. ${ }^{(6)} \mathrm{A}$ presença de uma descontinuidade altera a distribuição das tensões aplicadas no material, produzindo:

- elevação das tensões na proximidade da descontinuidade/ entalhe:

- introdução de um estado triaxial de tensões, mesmo que o componente tenha sido carregado apenas em tração (estado uniaxial);
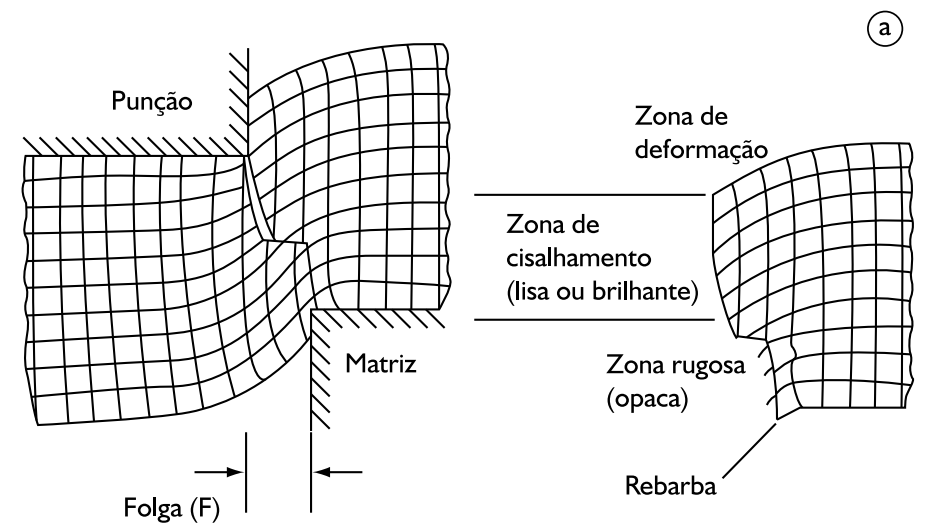

- endurecimento localizado com alta possibilidade de trincamento do material; e

- aumento localizado na taxa de deformação.

\section{CARACTERÍSTICAS DOS PROCESSOS DE CORTE}

\section{I Corte Mecânico: Melhoria das Bordas Cortadas}

No processo de corte por estampo ou por guilhotina as chapas são submetidas à ação de pressão exercida por um punção, uma lâmina ou uma navalha de corte. A superfície de uma peça metálica cortada desta forma normalmente apresenta quatro regiões, ilustradas na Figura $2^{(6,7)}$ :

- uma pequena região de deformação plástica pela compressão da navalha sobre a superfície da chapa (roll over zone);

- uma região lisa de cisalhamento, brilhante (burnish zone ou shiny), na qual a navalha efetivamente penetra no material;

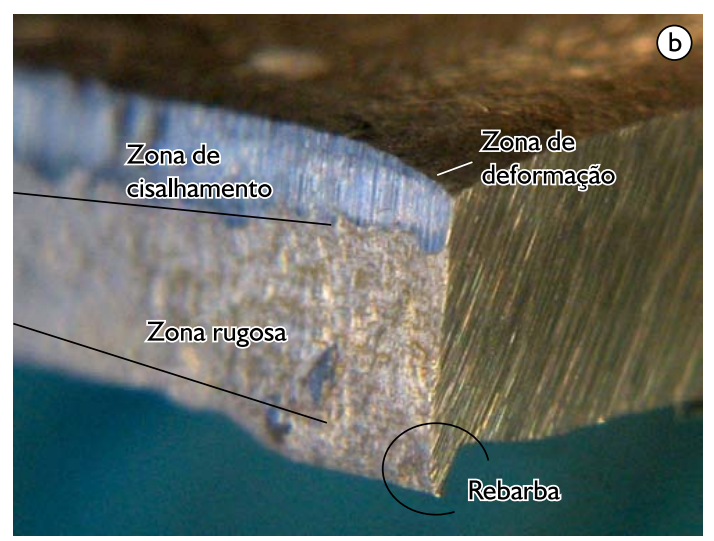

Figura 2. a e b) Vista uma superfície típica de uma peça cortada por estampo ou guilhotina. ${ }^{(5,6)}$ 
- uma região rugosa, opaca (matte ou fracture zone), na qual ocorre a ruptura final e assim também conhecida como região de "explosão";

- eventualmente, uma rebarba de arrancamento final (burr).

No final da década de 80 foram desenvolvidos e incorporados procedimentos de corte mecânico, conhecidos como fineblanking, que oferecem grande precisão e qualidade de borda. ${ }^{\left({ }^{(}\right)}$ Nesses casos, o processo de corte visa não gerar rugosidades ou rebarbas na superfície cortada, conforme exemplificado pela Figura 3. Esses procedimentos estão tornando-se comuns em algumas aplicações no País e requerem chapas com tolerâncias dimensionais mais restritas e um balanço adequado de propriedades (resistência/ductilidade) para essa aplicação.
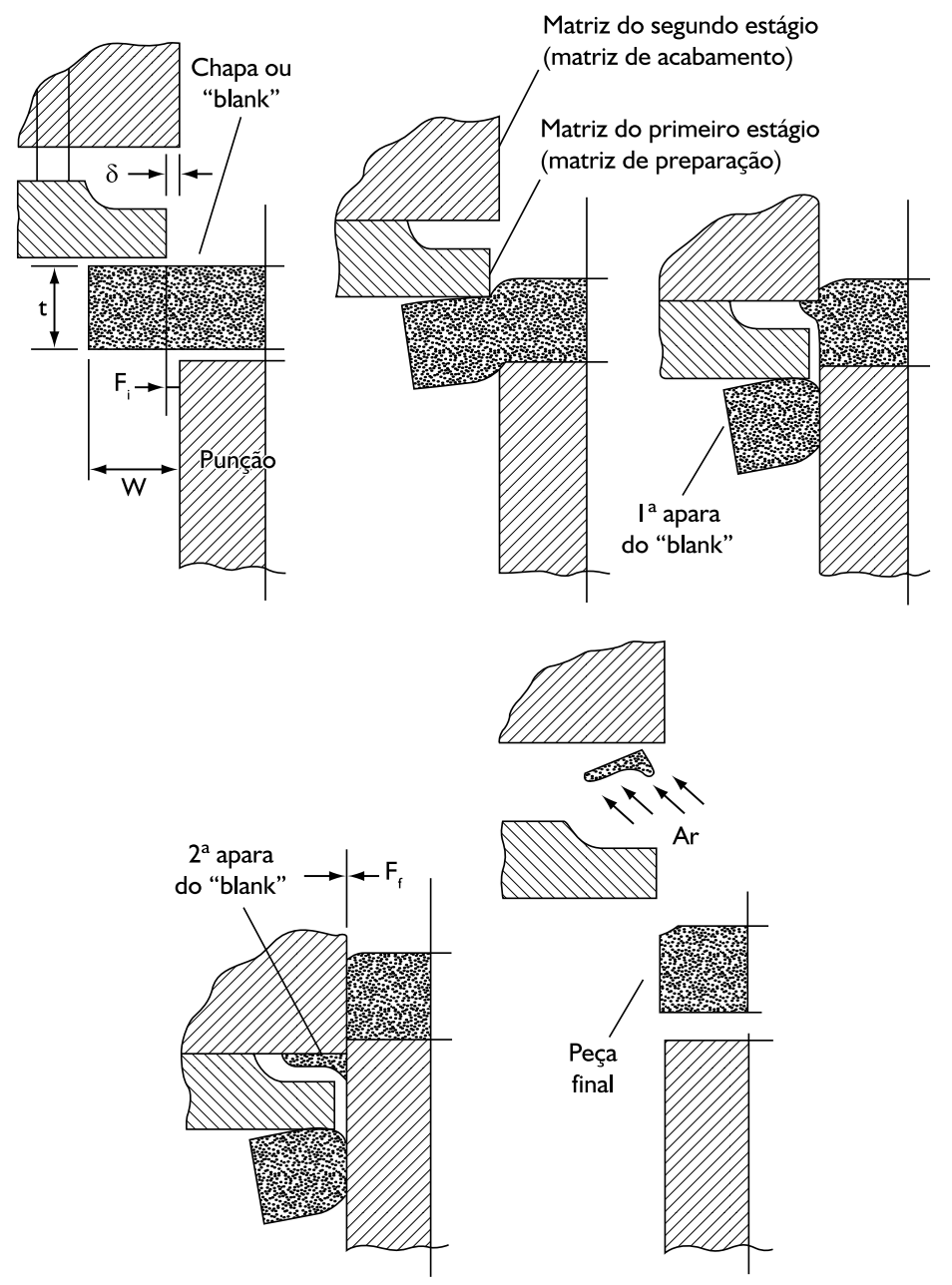

Figura 3. Exemplos de processo de corte por estampo visando a melhoria de acabamento superficial de peças cortadas (fineblanking). ${ }^{(8)}$

\subsection{Corte Térmico: o Avanço do Laser}

Os processos de corte que envolvem fusão localizada são por plasma, oxicorte e por laser. Cada um possui uma faixa de aplicações relativamente definida em função da espessura da peça a ser cortada. Esses processos são caracterizados por obter formas complexas em peças muito espessas (oxicorte ou plasma) ou em altas velocidades de produção aliadas à flexibilidade de tipos de corte (laser).
Dentre os processos de corte térmico, atualmente $\circ$ processo a plasma se destaca, ${ }^{(9)}$ uma vez que vem ganhando espaço no corte de chapas grossas na faixa de espessuras (e) de $5 \mathrm{~mm}$ a $40 \mathrm{~mm}$. Neste contexto, são cortados por oxicorte materiais mais espessos, acima de $32 \mathrm{~mm}$; pelo processo por laser, materiais mais finos, abaixo de $6 \mathrm{~mm}$.

No processo por laser, a energia elétrica é transformada em uma luz com um só comprimento de onda $(\lambda)$, que concentra energia de forma muito eficaz. O meio para formação do laser pode ser sólido (YAG) ou gasoso $\left(\mathrm{CO}_{2}, \mathrm{~N}_{2}\right.$ ou $\left.\mathrm{He}\right)$. Como nos demais processos de corte térmico, no corte por laser é empregado um gás de assistência, dentre os quais os mais utilizados são: o oxigênio, o nitrogênio ou o Ar comprimido. A definição da combinação entre $\circ$ gás de assistência e o metal cortado é feita conforme a espessura, a velocidade e a qualidade de corte necessárias para as peças a serem obtidas. (I)

A precisão do corte por laser dependerá de fatores adicionais: regulagem do equipamento, qualidade superficial da chapa, nível de tensão residual e composição química. Neste caso, a rugosidade do corte de chapas de aço aumenta com maiores teores de carbono, fósforo e molibdênio e a qualidade do corte é melhorada com menores teores de enxofre e silício. ${ }^{(10)}$ No caso do silício, esta é uma condição amplamente reconhecida e está ilustrada na Figura 4a.

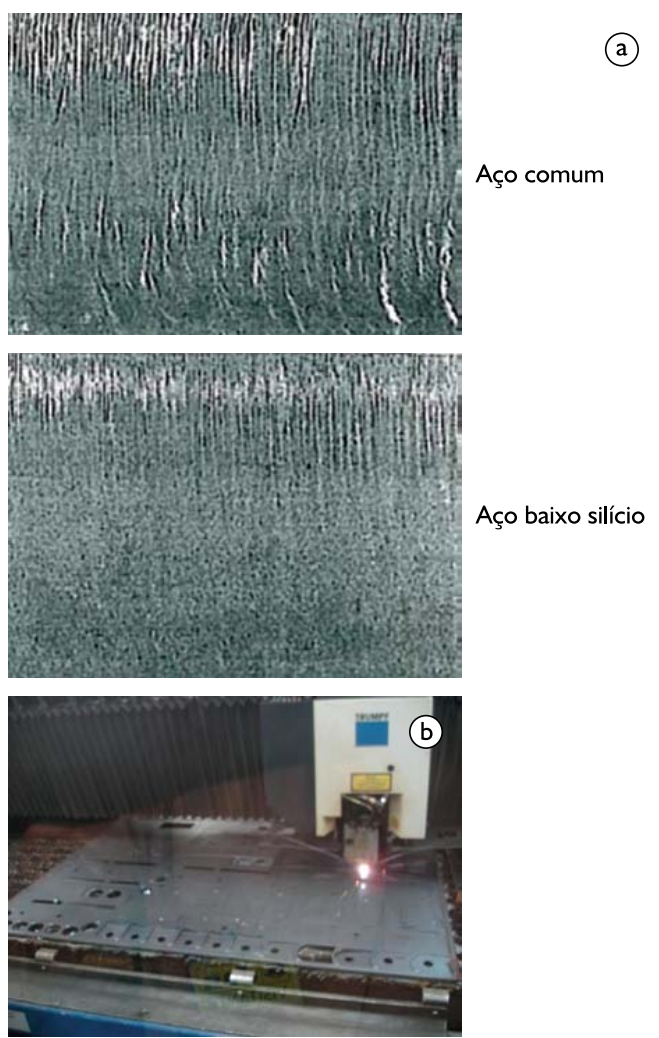

Figura 4. a) Aspecto da borda lateral de uma chapa de $\mathrm{I}, 0$ pol $(25,4 \mathrm{~mm})$ de dois aços diferentes ${ }^{(10)}$. b) Exemplo da operação de corte por laser com várias peças sendo obtidas de uma mesma chapa. 
O processo de corte por laser é o mais recomendado quando se necessitam peças com um melhor acabamento. $O$ corte por laser atinge o melhor nível de precisão do ângulo da borda, representado pelos níveis I ou 2, conforme previsto na norma ISO 9013.(11) O corte por laser também é a melhor opção para produzir peças de formas relativamente complexas e em quantidades variadas. Essa característica permite ao produtor racionalizar a produção, reduzindo as perdas por descartes, aproveitando o máximo da superfície da chapa, como ilustra a Figura 4b.

\section{MELHORIA NO DESEMPENHO NOS PROCESSOS DE CORTE E DOBRA}

\section{I Geometria do Dobramento e Ductilidade do Metal}

Chapas de aço podem apresentar alguma anisotropia gerada, por exemplo: por textura microestrutural (grãos achatados ou alongados em uma determinada direção), bandeamento ferrítico-perlítico (especialmente em aços com maior teor em manganês) ou mesmo pela eventual presença de inclusões alongadas em aços mais simples. Assim, é de primordial importância priorizar a operação de dobramento com o eixo de dobra orientado na direção transversal da laminação da chapa. As anisotropias presentes podem produzir trincas, especialmente quando o material apresenta uma borda mais rústica ou quando se emprega uma geometria de dobramento desfavorável com grande relação espessura/raio interno.(I) Assim, aços com ductilidade diferenciada são recomendados quando:

- é necessário realizar uma operação de dobramento mais crítica, com uma razão entre espessura e raio interno (e/Ri) maior que I,6;

- deve-se dobrar, na direção mais favorável, chapas de aços com maior resistência, especialmente com limite de escoamento maior que $350 \mathrm{MPa}$.

Uma melhor relação entre ductilidade e resistência é obtida durante a fabricação dos aços através de refino de grão, uso de elementos microligantes, diminuição da quantidade de elementos residuais indesejáveis (por exemplo, enxofre) e tratamento para alteração da geometria ou formato das inclusões (globulização). ${ }^{(12)}$ $\mathrm{Na}$ Tabela I estão comparados três grupos de aços, definidos de acordo com o Desempenho Relativo (DR) em dobramento. Esse desempenho é representado pela Equação I, cuja proposta é inspirada na metodologia dos Índices de Mérito (IM):(13)

$$
\mathrm{DR}=\frac{\sigma_{\mathrm{LE}}}{2 \mathrm{e}+\mathrm{b}}[\mathrm{MPa} / \mathrm{mm}]
$$

onde: $\left(\sigma_{\mathrm{LE}}\right)$ limite de escoamento em MPa; (b) calço de dobramento permissível (adimensional); e (e) espessura da chapa em $\mathrm{mm}$.

Esta equação expressa de forma algébrica - compromisso entre duas características dos aços para dobramento, permitindo comparar diretamente as melhores opções: aqueles que apresentam boa capacidade de dobramento possuem um $\mathrm{DR}$ acima de $20 \mathrm{MPa} / \mathrm{mm}$, na espessura referência de $5,00 \mathrm{~mm}$.

\subsection{Acabamento do Corte}

Os processos de corte oferecem características de acabamento diferentes entre si. Para peças que serão posteriormente conformadas é necessário escolher o processo de corte mais adequado. A Figura 5 ilustra o desempenho na deformação de uma chapa metálica contendo um furo de igual tamanho inicial, mas produzido por diferentes processos de corte em chapas de aço Complex Phase. ${ }^{(18)} \mathrm{O}$ acabamento superficial, ilustrado por imagens obtidas por Microscopia Eletrônica de Varredura (MEV), oferecido pelo processo de corte da peça, melhora o desempenho obtido em conformação, que pode ser quantificado pelo tamanho máximo da expansão que pode ser obtido a partir do furo inicial: quanto melhor o acabamento, maior o tamanho do furo final obtido após a conformação.

A borda oriunda de um corte mecânico comum, obtida por estampo não produzido por um processo de fineblanking, apresenta um lado mais "brilhante" ou "liso" (Figura 2). Assim, deve-se orientar essa região da peça na região de tração do dobramento, onde ocorrem alongamento e redução localizada de espessura (Figura I). Também é possível introduzir um chanfro na lateral da chapa cortada, de maneira a

Tabela I. Exemplos de qualidades de aço e suas características mecânicas

\begin{tabular}{|c|c|c|c|c|}
\hline Tipo de aço & $\begin{array}{c}\text { Qualidade } \\
\text { (norma e grau aplicável) }\end{array}$ & $\begin{array}{c}\text { Calço de dobramento } \\
\text { a } 180^{\circ} \text { (b) }\end{array}$ & $\begin{array}{l}\text { Limite de escoamento } \\
\left(\sigma_{\mathrm{LE}}\right)\end{array}$ & $\begin{array}{l}\text { Desempenho relativo } \\
\text { (Equação I; e }=5,0 \mathrm{~mm}\end{array}$ \\
\hline \multirow{3}{*}{$\begin{array}{c}\text { Estruturais comuns } \\
\left(\mathrm{DR}_{\text {Méd }}=15 \mathrm{MPa} / \mathrm{mm}\right)\end{array}$} & ASTM AI0II SS36 TI(14) & $1,5 \cdot e^{(*)}$ & $\geq 250 \mathrm{MPa}$ & $14 \mathrm{MPa} / \mathrm{mm}$ \\
\hline & Al0II SS50 & $2,5 \cdot e^{(*)}$ & $\geq 340 \mathrm{MPa}$ & $15 \mathrm{MPa} / \mathrm{mm}$ \\
\hline & AIOII HSLAS $50 \mathrm{CI}^{(14)}$ & $2,0 \cdot e^{(*)}$ & $\geq 340 \mathrm{MPa}$ & $17 \mathrm{MPa} / \mathrm{mm}$ \\
\hline \multirow{3}{*}{$\begin{array}{l}\text { Estruturais com boa } \\
\text { conformabilidade } \\
\left(\mathrm{DR}_{\text {Méd }}=20 \mathrm{MPa} / \mathrm{mm}\right)\end{array}$} & NBR 6655 LN24(15) & $0,5 \cdot e$ & $\geq 240 \mathrm{MPa}$ & $19 \mathrm{MPa} / \mathrm{mm}$ \\
\hline & ASTM AIOI I HSLAS 50 C2 (14) & $\mathrm{I}, 5 \cdot \mathrm{e}^{(*)}$ & $\geq 340 \mathrm{MPa}$ & $19 \mathrm{MPa} / \mathrm{mm}$ \\
\hline & ASTM AI0II HSLAS-F50 & $1,0 \cdot e^{(*)}$ & $\geq 340 \mathrm{MPa}$ & $23 \mathrm{MPa} / \mathrm{mm}$ \\
\hline \multirow{3}{*}{$\begin{array}{l}\text { Estruturais com ótima } \\
\text { conformabilidade } \\
\left(\mathrm{DR}_{\text {Méd }}=35 \mathrm{MPa} / \mathrm{mm}\right)\end{array}$} & NBR 6656 LNE260(16) & $0,0 \cdot e$ & $\geq 260 \mathrm{MPa}$ & $26 \mathrm{MPa} / \mathrm{mm}$ \\
\hline & NBR 6656 LNE380(16) & $0,0 \cdot e$ & $\geq 380 \mathrm{MPa}$ & $38 \mathrm{MPa} / \mathrm{mm}$ \\
\hline & USI LN $500^{(17)}$ & $0,5 \cdot \mathrm{e}$ & $\geq 500 \mathrm{MPa}$ & $40 \mathrm{MPa} / \mathrm{mm}$ \\
\hline
\end{tabular}

$\mathrm{e}=$ Espessura nominal do material. ${ }^{(*)}$ Neste caso, a norma não oferece esta garantia e sim uma recomendação. 

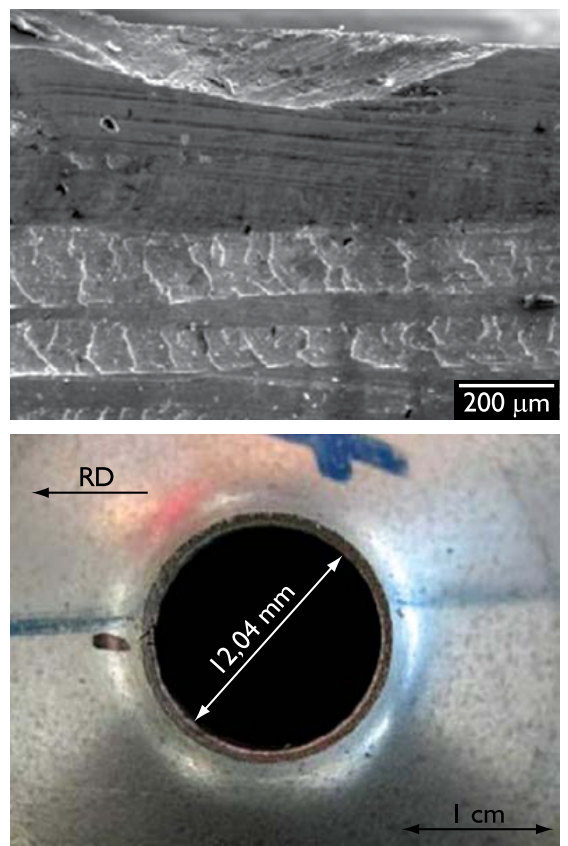

Estampagem ( $12,0 \mathrm{~mm})$
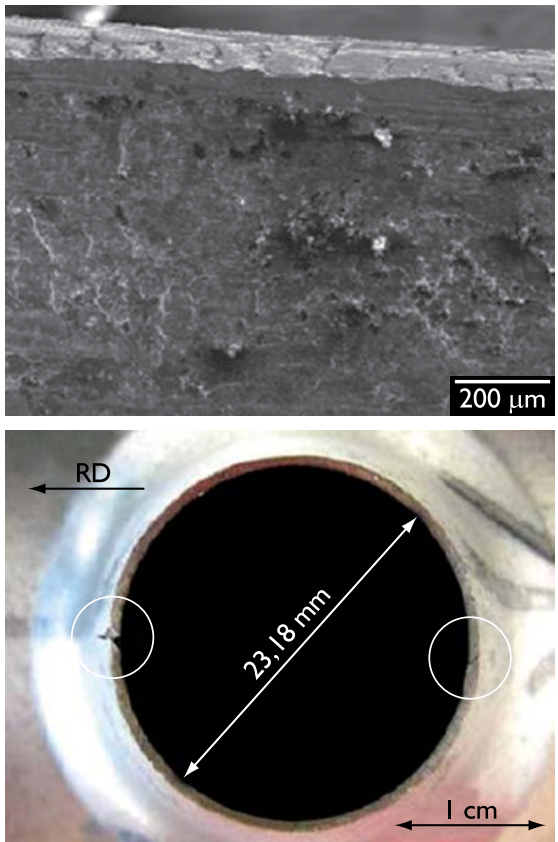

Perfuração ( $23,2 \mathrm{~mm})$
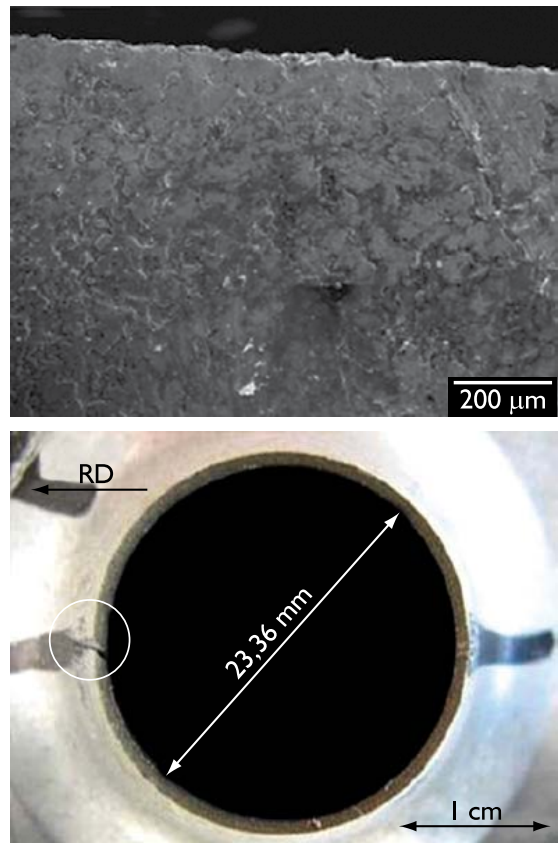

Corte $\mathrm{a}$ arco $(23,4 \mathrm{~mm})$

Figura 5. Desempenho no ensaio de expansão de furo de um aço processado por três processos diferentes de corte, cujos acabamentos na borda estão ilustrados pelas imagens obtidas por MEV.(18)

melhorar a sua qualidade superficial, reduzir ou eliminar os pontos de concentração de tensão e assim melhorar o desempenho do material. A Figura 6.a ilustra um caso prático no qual a conformação foi prejudicada devido às rebarbas de corte da peça.

A Figura $6 \mathrm{~b}$ ilustra o acabamento de um corte por laser feito para obter blanks (pré-formas) de uma ferramenta a partir de uma chapa de aço SAE 1045, com 0,25\%Si e $9,50 \mathrm{~mm}$ de espessura. Pode-se notar, na parte inferior do corte, uma série de imperfeições similares a rebarbas de corte por estampo e que a superfície da chapa de aço possui a formação de carepas conhecidas como "siliciosas", basicamente constituídas por faialita $\left(\mathrm{Fe}_{2} \mathrm{SiO}_{4}\right)$ e hematita $\left(\mathrm{Fe}_{2} \mathrm{O}_{3}\right)$. A presença do silício nos aços cortados por laser passa a ser cada vez mais prejudicial à qualidade do corte com o aumento da espessura das chapas. Recomenda-se, para um bom corte, silício máximo de $0,05 \%$ para chapas com espessuras acima de $5 \mathrm{~mm}$.

\subsection{Tensões Residuais}

Tensões residuais ocorrem em chapas de aço, especialmente se forem obtidas de bobinas de aço. Neste caso, as chapas possuíam, no mínimo, a curvatura original da bobina que foi retirada por desempeno feito a frio. Essa operação pode gerar tensões residuais, tais como as ilustradas na Figura 7a. $O$ nível de tensão necessária para $\circ$ desempeno aumenta com $\circ$ grau de resistência do material, daí necessitando de equipamentos cada vez mais sofisticados e rígidos ou robustos ${ }^{(19)}$ para fazer o devido aplainamento do material. O grande problema que pode ocorrer, com um nível mais elevado de tensões residuais,é a distorção (empeno ou mesmo levantamento) das peças que estão sendo cortadas, como ilustrado pela Figura $7 \mathrm{~b}$.

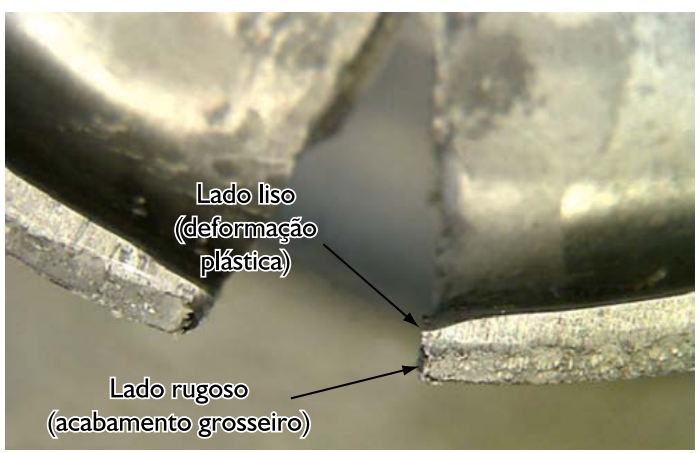

(a)

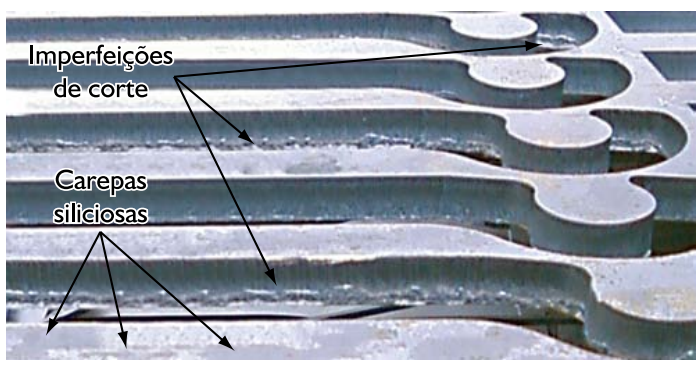

(b)

Figura 6. a) Detalhe de uma peça automobilística que rompeu na conformação com a indicação que o lado rugoso da peça estava localizado na região mais crítica (tração); e b) resultado do corte por laser de uma chapa de aço $\mathrm{SAE}$ 1045 (com 0,25\%Si e $9,50 \mathrm{~mm}$ de espessura).

Neste caso, pode-se trabalhar em quatro frentes, de acordo com a sequência de possibilidades de melhorias listadas a seguir: 

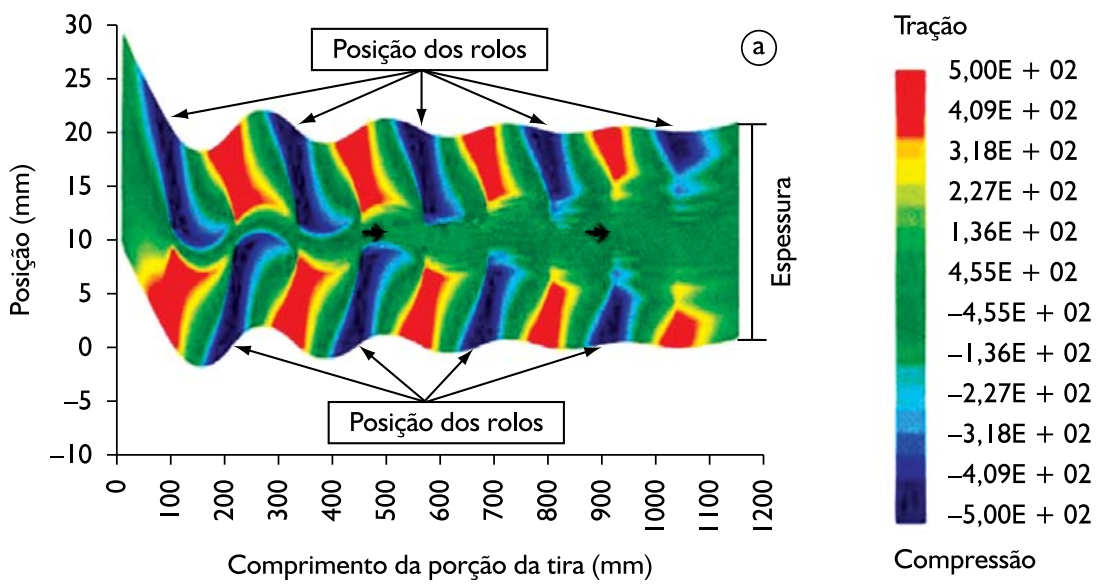

Figura 7. a) Distribuição de tensões durante o processo de aplainamento(19); e b) desprendimento natural de uma parte cortada por laser de uma chapa com presença de tensão residual.

- alterar o projeto de liga do aço e o processo de laminação de forma a minimizar as condições que levam ao tensionamento do material;

- alterar o processo de desempeno das chapas, para introduzir o mínimo de tensões residuais, utilizando-se desempenadeira mais rígida (robusta), ou do tipo aplainadora (levelling), ou mesmo desempenadeira sob tensão;

- realizar o corte em etapas, deixando-se pequenos pontos de união entre a peça cortada e a chapa de forma que o processo de separação possa ser terminado depois, fora da máquina de corte a laser;

- trocar o tipo de produto de chapa oriunda de bobinas (produzidas por laminador contínuo) para chapas oriundas de laminador reversível (que não foram bobinadas anteriormente).

\section{CONCLUSÕES}

O histórico de experiências, análises e ações para melhoria da utilização dos produtos da Usiminas-Cubatão, aqui apresentados, levam às seguintes conclusões, que se tornam recomendações importantes para o corte e dobramento de chapas:

- o processo de corte pode ser variado, assim como a influência sobre o processo de dobramento posterior;

- o resultado com a peça cortada depende do material, mas também de como esta foi cortada e manuseada; e

- práticas diferenciadas, como as descritas neste trabalho, podem ser adotadas de forma a melhorar o desempenho em corte e dobramento de produtos planos de aço, aplicáveis para casos e condições particulares.

\section{REFERÊNCIAS}

I BENSON, S. D. Lasers, punches, press brakes \& shears. Asma: LLC Interactive Textbook, 2002. I CD.

2 MORAIS, W. A.; BORGES, H. C. Condições técnico-economônicas para viabilizar o emprego de aços planos de elevada resistência mecânica em aplicações práticas. Tecnologia em Metalurgia, Materiais e Mineração, v. 6, n. I, p. I-6, jul.-set. 2009.

3 MAGNABOSCO, A. S. Resistência mecânica $\times$ conformabilidade. In: MORAIS, W. A.; MAGNABOSCO, A. S; NETTO, E. B. M. Metalurgia física e mecânica aplicada. 2. ed. São Paulo: ABM, 2009. p. 48I-500.

4 CALLISTER, W. D. Materials science and engineering. 4. ed. New York: John Wiley \& Sons, 1997.

5 KESTENBACH, H. J.; CAMPOS, S. S. Contribuição da precipitação interfásica à resistência mecânica em tiras a quente. Tecnologia em Metalurgia e Materiais, v. I, n. I, p. 28-33, jul. 2004.

6 ZELT, A. L.; KERNS, H.; BRESNAHAN, F. Slitting knives and setups. In: THEIS, H. E. Handbook of metalforming process. New York: Marcel Dekker, 1999. Cap. 4., p.I I7-44.

7 KONIECZNY, A.; HENDERSON, T. On formability limitations in stamping involving sheared edge stretching. In: STEEL INNOVATIONS, FATIGUE RESEARCH, SHEET/HYDRO/GAS FORMING TECHNOLOGY \& ADVANCED HIGH STRENGTH STEEL DEVELOPMENT, 2007, Detroit. Proceedings... Warrendale: SAE International, 2007. p. $4 I-50$.

8 KONDO, K. Recent developments of shearing in Japan. International Journal of Machine Tools and Manufacture, v. 29, n. I, p. 29-38, 1989. 
9 LIMA, E. G. Corte a plasma. Revista da Soldagem, v. 2, n. 9, p. 18-26, 2006. Disponível em: <http://www.baw.com.br/ images/products/sup_3_Artigo_Corte_Plasma.pdf >. Acesso em: I3 abr. 2010.

10 MANOHAR, M. CO ${ }^{2}$ laser beam cutting of steels: material issues. Journal of Laser Applications, v. 18, n. 2, p. 10I-2, 2006.

II INTERNATIONAL ORGANIZATION FOR STANDARDIZATION. ISO 9013 - Welding and allied processes: quality classification and dimensional tolerances of thermally cut (oxygen/fuel gas flame) surfaces. Geneva, 2002.

12 MORAIS, W. A. Análise das relações entre as características dos aços e sua tenacidade. In: CONGRESSO ANUAL DA ABM, 65., 2010, Rio de Janeiro, RJ. Anais... São Paulo: ABM, 20I0. p. 2545-56.

13 ASHBY, M. F. Materials selection in mechanical design. Oxford: Pergamon Press, 1993.

I4 ASTM INTERNATIONAL. ASTM. A I0I I/A I0I IM - Specification for steel, sheet and strip, hot-rolled, carbon, structural, high-strength low-alloy and high-strength low-alloy with improved formability, and ultra-high strength. West Conshohocken, 2010.

I5 ASSOCIAÇÃO BRASILEIRA DE NORMAS TÉCNICAS. NBR 6655 - Chapas de aço com características melhoradas de propriedades mecânicas, conformabilidade e soldabilidade. Rio de Janeiro, 1984.

16 ASSOCIAÇÃO BRASILEIRA DE NORMAS TÉCNICAS. NBR 6656 - Chapas e bobinas de aço acalmado com características especiais de propriedades mecânicas, conformabilidade e soldabilidade. Rio de Janeiro, 2008.

17 USIMINAS. SX-3900-Q-8NT0027 - Norma de produtos corporativa. Belo Horizonte, 2010.

I8 KARELOVA, A. et al. Influence of the edge conditions on the hole expansion property of dual-phase and complex-phase steels.In: MATERIALS SCIENCE AND TECHNOLOGY,, 7., 2007, Detroit. Proceedings... Tokyo: AIST, 2007. p. I59-70.

19 HARTUNG, H. G.; JAENECKE, M.; SASSE, C. New shearing line for a more efficient production of plates with few inherent stress. Stahl und Eisen, v. I29, n. 10, p. 55-9, Oct. 2009.

Recebido em: $13 / 10 / 2010$

Aceito em: 27/01/2011 